\title{
ANÁLISIS DE LA VEGETACIÓN ARBÓREA EN LA PROVINCIA FLORÍSTICA DE TEHUACÁN-CUICATLÁN
} VÍCTOR JARAMILLO LUQUE* y FRANCISCO GONZÁLEZ MEDRANO**

\section{RESUMEN}

Se hace un análisis de la vegetación arbórea de una porción del Valle de TehuacánCuicatlán a lo largo de las laderas a sotavento de las Sierras de Juárez y Zongolica (Sierra Madre Oriental), siguiendo un gradiente altitudinal. Para el análisis se utilizaron técnicas de análisis multivariado tanto de ordenación (análisis de componentes principales y promediación recíproca) como de clasificación (análisis de información con el programa CENOSIS 2). Los resultados permiten la definición de “grupos" identificables con tipos de vegetación definidos desde un punto de vista fisonómico-estructural. Además, el análisis realizado muestra que la vegetación arbórea de la zona de estudio presenta una variabilidad y heterogeneidad que estudios fisonómico-descriptivos anteriores no habían detectado. Asimismo se discute la importancia que tienen la perturbación humana y otros factores (p. ej. interacciones bióticas) al afectar la estructura y composición de las comunidades vegetales de la zona de estudio.

Se comparan también los alcances de cada técnica de análisis, mostrándose la mayor eficiencia del método de clasificación cuando se manejan niveles altos de heterogeneidad vegetacional. Se proponen, finalmente algunas cuestiones que se juzgan interesantes para futuras investigaciones.

\begin{abstract}
The arboreal vegetation of a portion of the Tehuacán-Cuicatlán Valley is analyzed. An altitudinal gradient was followed along the leeward slopes of the Sierras de Juárez and Zongolica (Sierra Madre Oriental). Multivariate analysis techniques, ordination (principal component analysis and reciprocal averaging) and classification (informationanalysis program CENOSIS 2) were used. The results allow the definition of "groups"

*Este trabajo es parte de la tesis profesional presentada en la Facultad de Ciencias, UNAM, para optar por el título de biólogo; actualmente trabajando en el herbario de COTECOCA, SARH. Recibido para publicación en noviembre de 1983.

**Instituto de Biología, UNAM.
\end{abstract}


identifiable with vegetation types defined from a physiognomic-structural view point; however, the analysis performed shows that the arboreal vegetation of the study area possesses a variability and heterogeneity not detected by previous descriptivephysiognomic studies. The importance of human disturbance and other factors (e.g. biotic interactions) affecting the structure and composition of the plant communities of the study area is discussed.

The analysis techniques are compared with regards to their effciency in handling high levels of vegetational heterogeneity, being the clasification technique the most efficient one. Finally, some considerations jugded interesting for future investigations are proposed.

\section{INTRODUCGIÓN}

La utilización de los métodos numéricos descriptivos, ordenación y clasificación, en los estudios fitosociológicos tuvo un desarrollo y crecimiento notables a partir de la década de 1950-1960. Los trabajos de ordenación producidos por Brown y Curtis (1952), Goodall (1954), Curtis (1955) y Bray y Curtis (1957), así como los de clasificación realizados por Goodall (1953) y Williams y Lambert (1959) forman la base a partir de la cual proliferan, en la siguiente década, un número cada vez mayor de técnicas numéricas para al análisis de la vegetación (Dagnelie, 1960, en Kershaw, 1973; Austin y Orloci, 1966; Orloci, 1966; Williams y Lambert, 1961; Williams, et al., 1966; Lambert y Williams, 1966). Los logros alcanzados fueron el fruto tanto de la introducción de métodos de muestreo y clasificación objetivos y cuantitativos, como de la aplicación de métodos de análisis multivariado y de la reconsideración teórica del concepto individualista de las comunidades postulado por Ramensky y Gleason entre 1924 y 1926.

De acuerdo con Austin (1972) el uso de estas técnicas de análisis tiene tres propósitos básicos:

a) simplificar datos multivariados, es decir, hacer más accesible al análisis la interrelación de las múltiples variables que se consideran al estudiar la vegetación,

b) ayudar en la generación de hipótesis que permitan investigar con detalle los patrones de variación observados, la distribución de las especies, la estructura de las comunidades, etc., y,

c) definir el dominio, esto es, definir el tipo de vegetación en que se pueden llevar a cabo experimentos que nos permitan llegar a niveles más explicativos en cuanto a la naturaleza de la vegetación, así como los probables límites de extrapolación de dichos resultados experimentales.

Aunque intrínsecamente los fundamentos teóricos y los objetivos de la ordenación y la clasificación son diferentes, numerosos autores (Lambert y Dale, 1964; Anderson, 1965; Webb et al., 1967; McIntosh, 1967; Greig-Smith et al., 1967; Maarel, 1969; Goodall, 1970, 1978; Whittaker, 1970, 1975; Mueller-Dombois y Ellenberg, 1974) postulan que ambas técnicas son complementarias, pudiendo producir información valiosa cuando ambas son aplicadas a un mismo estudio de vegetación.

Sin embargo, el enfoque más utilizado desde los inicios de la fitosociología ha sido el de la clasificación de comunidades, y aunque las clasificaciones se hacían con criterios más o menos subjetivos, actualmente, con la introducción de los métodos numéricos se ha tratado de producir clasificaciones más objetivas.

Los estudios fitosociológicos y entre ellos los que utilizan los métodos numéricos 
descriptivos (ordenación y clasificación), son importantes en tanto que proveen un marco de referencia con el cual correlacionar cambios de morfología, vitalidad, dispersión y fenología de las plantas que son determinados por cambios en la composición de la comunidad (Curtis y McIntosh, 1951; Bray y Curtis, 1957; Lambert y Dale, 1964). Es en este mismo sentido que Curtis y McIntosh (op. cit.) plantean la necesidad de establecer las relaciones sociológicas y numéricas de las especies para que los estudios autoecológicos tengan su máximo significado.

El objetivo del presente trabajo es hacer un análisis de la vegetación arbórea en la parte oriental y norte del Valle de Tehuacán-Cuicatlán, mediante la aplicación de técnicas de análisis multivariado: dos de ordenación, análisis de componentes principales - ACP - (Ezcurra, 1978) y promediación recíproca - PR - (Hill, 1973), y una de clasificación - CENOSIS 2- (Ezcurra y Equihua, manuscrito no publicado) a lo largo de un gradiente altitudinal.

\section{MATERIALES Y MÉTODOS}

\section{Descripción de la zona de estudio}

La zona de estudio (Fig. 1) se encuentra enclavada dentro de lo que se conoce como la Provincia Florística de Tehuacán-Cuicatlán (Rzedowski, 1978). Se localiza entre los $17^{\circ} 48^{\prime}$ y $18^{\circ} 58^{\prime}$ de latitud norte y los $97^{\circ} 03^{\prime}$ y $97^{\circ} 43^{\prime}$ de longitud oeste, siguiendo una dirección sureste-noroeste por la Sierra de Juárez y Zongolica, en la parte sureste del estado de Puebla y noroeste del de Oaxaca. Desde el punto de vista fisiográfico abarca el Valle de Cuicatlán y el Valle de Tehuacán que son considerados por Miranda (1948) como parte de la Cuenca Alta del Papaloapan.

Siguiendo los criterios de García (1973), el clima de la zona corresponde al tipo semiárido $\left(\mathrm{BS}_{0}\right.$ y $\left.\mathrm{BS}_{1}\right)$, con condiciones de temperatura cálida y semicálida; régimen de lluvias de verano con canícula y con poca a extremosa oscilación de temperatura. Este clima se debe fundamentalmente al efecto de sombra de lluvia que producen las Sierras de Juárez y Zongolica.

El Valle de Tehuacán es recorrido principalmente por el río Salado que sigue su curso hacia Oaxaca y en Quiotepec se une con el Río Grande que trae aguas del Valle de Cuicatlán. De esta unión se forma el Río Santo Domingo, afluente del Papaloapan, el cual desemboca finalmente al Golfo de México.

Desde el punto de vista geológico (López Ramos 1974, 1979; Anónimo, 1981) la porción de la Sierra de Juárez que llega hasta Quiotepec, así como parte de la de Zongolica, presentan afloramientos de rocas metamórficas (esquistos) del Paleozoico y en las partes bajas afloramientos de sedimentos del Terciario continental y areniscas y conglomerados del Cuaternario. De Teotitlán del Camino hacia Tehuacán se presentan afloramientos del Precámbrico con esquistos y gneises indiferenciados, así como lutitas y areniscas del Jurásico inferior. En los alrededores de Tehuacán existen afloramientos de rocas calizas del Cretácico Superior e Inferior.

Los suelos que se presentan en la región (Anónimo, 1981) corresponden a una combinación de luvisol vértico, litosol y regosol eútrico en la región de Cuicatlán. El faeozem háplico se encuentra en la región de Quiotepec y hacia Tehuacán se presenta una combinación de litosol, rendzinas y faeozem háplico.

\section{Metodología}

Se realizaron salidas al área con el objeto de hacer un reconocimiento general y 


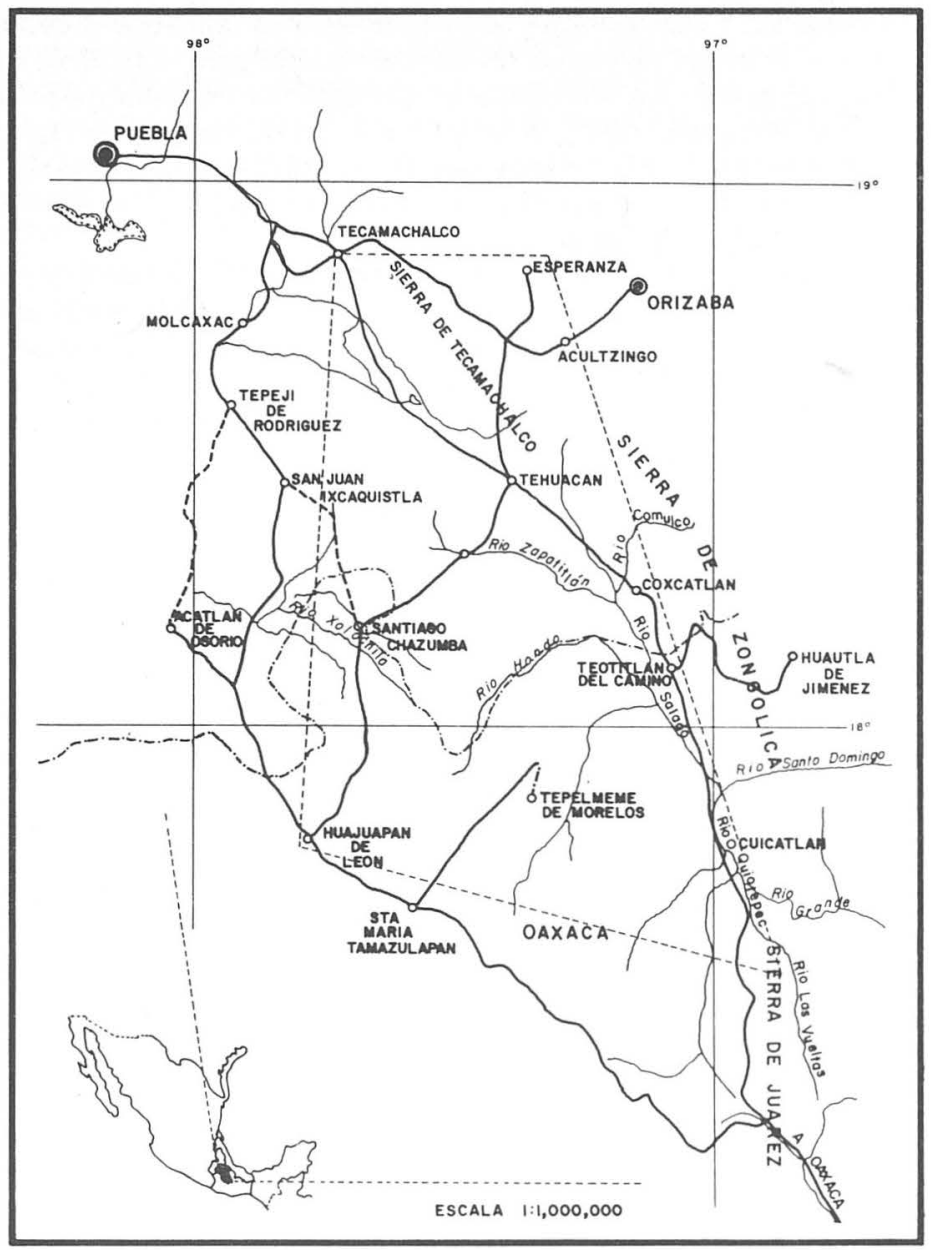

Fig. 1. La Provincia Florística de Tehuacán-Cuicatlán.

colectar material botánico. La zona de trabajo se escogió en función de la posibilidad de estudiar la variación de la vegetación siguiendo un gradiente altitudinal que va desde aproximadamente los $500 \mathrm{msnm}$ en la zona de Quiotepec y Cuicatlán hasta los 1670 msnm en el área de Tehuacán.

La ubicación de los sitios de muestreo se hizo de manera sistemática por diversas razones y siguiendo los criterios de varios autores (Lambert y Dale, 1964; Goodall, 1970; Kershaw, 1973). Estos son:

a) no se requería de una estimación de la media y del error; 


\section{ANÁLISIS DE LA VEGETACIÓN DE TEHUACÁN-CUICATLÁN}

b) se requería la misma cantidad de información sobre cada parte del área bajo estudio manteniendo constante la distancia entre las muestras;

c) se requería obtener datos de abundancia de las especies en un gradiente ambiental.

Se realizó un muestreo de las laderas a sotavento de las Sierras de Juárez y Zongolica, cada $2 \mathrm{~km}$ desde las inmediaciones de Cuicatlán hasta Tehuacán (Fig. 2).

Para la evaluación de las especies en cada sitio de muestreo se utilizó el método de muestreo de cuadrantes centrados en un punto (Cottam y Curtis, 1956). Este méto-

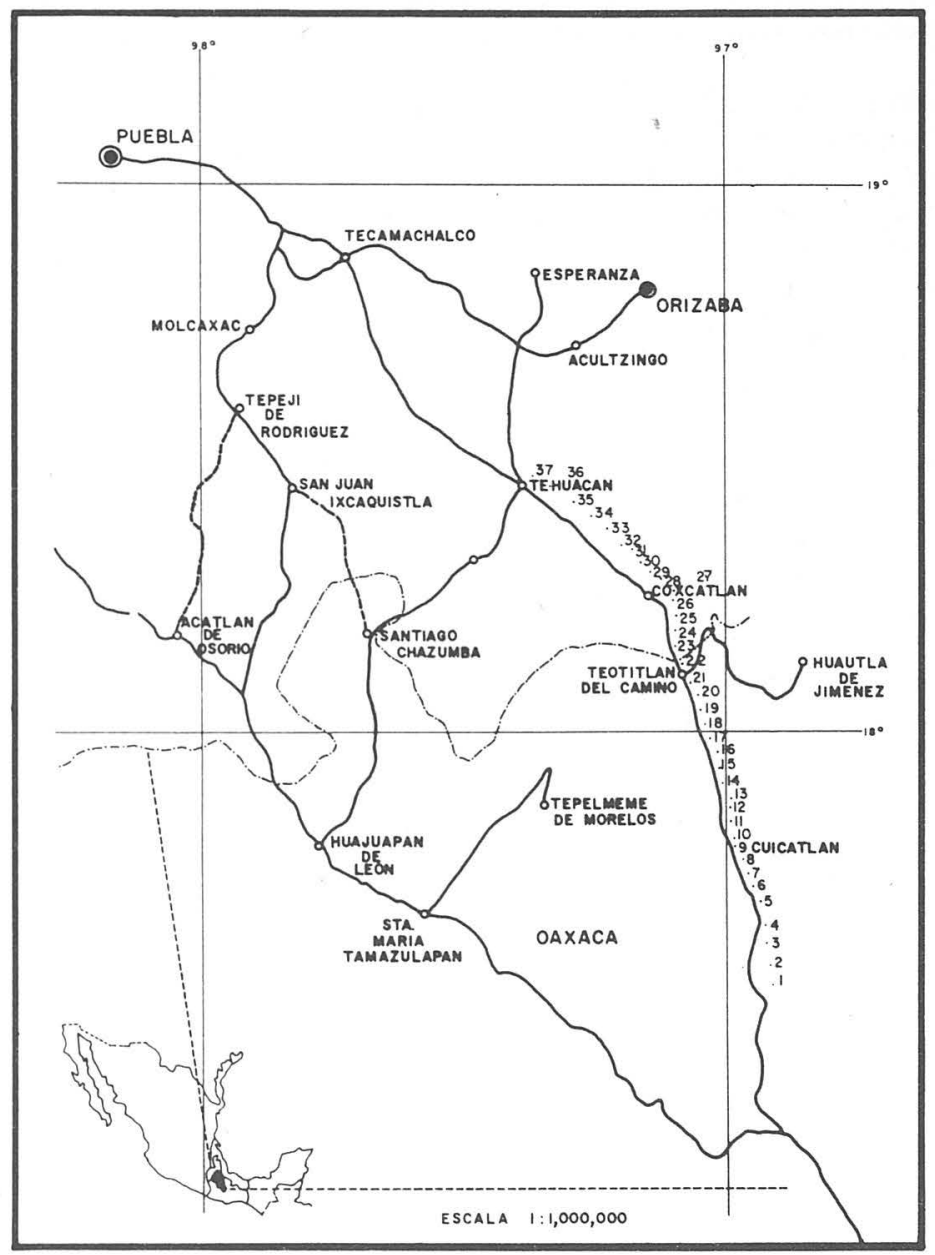

Fig. 2. Ubicación de los sitios de muestreo en la zona de estudio. 
do fue aplicado utilizando dos cuerdas de $60 \mathrm{~m}$ de largo marcadas cada $3 \mathrm{~m}$ que se tiraban perpendicularmente a la ladera, separadas $10 \mathrm{~m}$ una de otra y paralelas entre sí. Se tenían así 41 puntos de muestreo. Siguiendo las observaciones de Cottam y Curtis (1956) en cuanto a la necesidad de muestrear un número no menor de 20 puntos, de introducir una técnica aleatoria para hacer más objetivo el muestreo y por conveniencia práctica de cálculo, se sorteaban 25 puntos al azar para ser muestreados. De esta forma, dado que el muestreo se realizó para dos estratos, se muestreaban 200 individuos por sitio. Sin embargo, sólo el estrato arbóreo es considerado en el presente trabajo.

Además de la forma de crecimiento se consideró la altura de 2 m o más para escoger los árboles a muestrear ya que los elementos del bosque tropical caducifolio con frecuencia presentan troncos retorcidos, ramificándose a corta altura o casi desde la base, haciendo muy difícil definir precisamente las formas de crecimiento (Rzedowski, 1978).

En cada punto de muestreo se tomaron los siguientes datos: a) identificación de la planta (familia, género o especie); b) distancia del punto al centro de ramificación o del tronco según el caso; c) radio de cobertura de la planta; d) altura de la planta. En cada sitio se registraba: a) altitud sobre el nivel del mar; b) orientación de la ladera; c) estimación visual de pedregosidad y afloramientos de roca madre; d) estimación cualitativa de la pendiente de la ladera; e) el grado aparente de perturbación.

Para cada especie y por sitio se calculó: a) densidad: expresada en número de individuos por hectárea; b) cobertura promedio $\left(\mathrm{m}^{2}\right)$; c) frecuencia; d) dominancia: expresada como el producto de la densidad por la cobertura $\left(\mathrm{m}^{2} / \mathrm{ha}\right)$.

Todos los cálculos se hicieron en términos absolutos siguiendo el ejemplo citado por Mueller-Dombois y Ellenberg (1974), y se llevaron a cabo en la computadora del Departamento de Bioestadística del Instituto Nacional de Cardiología.

Para el análisis de los datos se utilizaron tres técnicas: dos de ordenación y una de clasificación. Las de ordenación corresponden al análisis de componentes principales (Ezcurra, 1978) y promediación recíproca (Hill, 1973). La de clasificación corresponde al análisis de información con el programa CENOSIS-2 (Ezcurra y Equihua, manuscrito no publicado).

$\mathrm{El}$ análisis de componentes principales fue aplicado a datos de dominancia de las especies ordenando los censos en función de los valores de las especies presentes (atributos) en ellos. La promediación recíproca se hizo para datos tanto de dominancia de especies como para datos de presencia-ausencia obteniéndose ordenaciones por censos y por especies. De éstas solo las ordenaciones por censos fueron utilizadas en el análisis. El método de clasificación utilizado es un método divisivo, monotético y está diseñado para datos de presencia-ausencia de especies. Los programas de análisis fueron aplicados en la computadora de la Escuela de Biología Vegetal en Bangor, Gales del Norte.

\section{RESULTADOS}

La Fig. 3 muestra los resultados de la clasificación. Esta se detuvo arbitrariamente (ver Lambert y Williams, 1966; Ezcurra y Equihua, manuscrito no publicado) al formarse 10 grupos. Se produjeron arreglos de censos en los cuales podemos definir "grupos" que en algunos casos corresponden a los tipos de vegetación fisonómicoestructurales tanto de Miranda y Hernández X. (1963) como de Rzedowski (1978). 


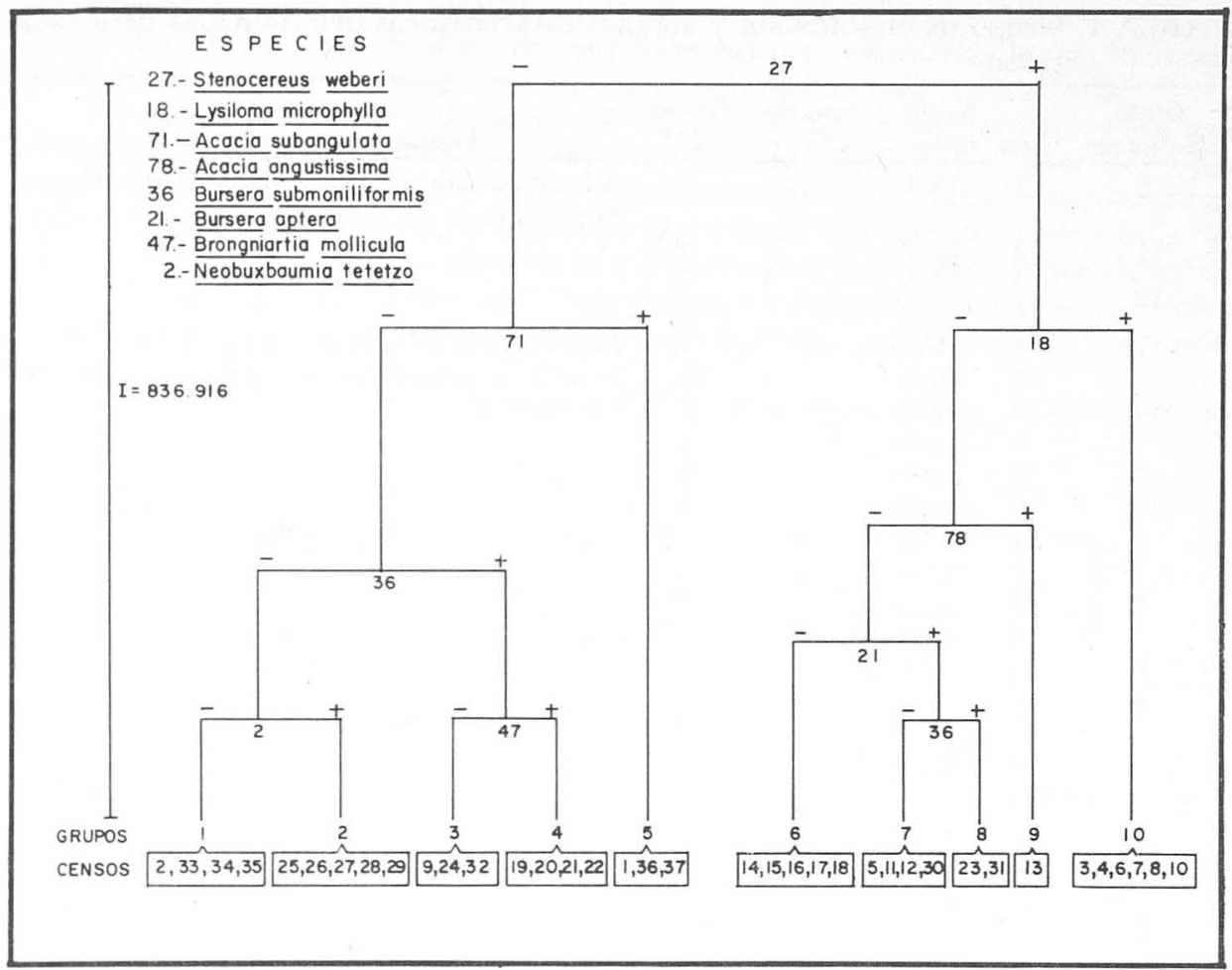

Fig. 3. Dendrograma de la clasificación de vegetación arbórea con el programa CENOSIS $2 .+=$ presente $;-=$ ausente; $I=$ información total.

La vegetación de cada censo y el grupo en que quedó clasificado se resumen en la tabla 1. Los "grupos" que se pueden definir a partir del análisis de clasificación son: las tetecheras de Neobuxbaumia tetetzo (grupo 2); la selva baja caducifolia con dos variantes: i) la de Bursera spp., Cercidium plurifoliolatum, Cyrtocarpa procera y Ceiba parvifolia sin Stenocereus weberi (grupos 3 y 4) y, ii) la de Lysiloma microphylla con Stenocereus weberi y algunas de las especies anteriores (grupo 10); los matorrales perturbados con Acacia cochliacantha (grupo 6) y matorrales también perturbados, pero de Ipomoea wolcottiana y $A$. cochliacantha (grupo 1).

Se produjo la clasificación errónea de algunos censos, que ya Lambert y Williams (1966) habían mencionado como una de las fallas de los métodos divisivos y monotéticos como el utilizado en el presente trabajo. En esta situación se encuentra el censo 2 que a pesar de que por su composición y fisonomía corresponde a las selvas bajas de Lysiloma (grupo 10), fue colocado en el grupo 1 por la ausencia de Stenocereus weberi. En el caso del censo 30 (grupo 7) y el 23 y 31 (grupo 8) la presencia de $S$. weberi evita su ubicación en los grupos en que por su fisonomía y composición corresponderían. 
TABLA 1. Grupo de clasificación y algunas características determinadas para cada censo

\begin{tabular}{|c|c|c|c|c|c|}
\hline $\begin{array}{c}\text { Grupo } \\
\text { clasificación }\end{array}$ & Censo & $\begin{array}{l}\% \text { spp. }{ }^{*} \\
\text { espinosas }\end{array}$ & $\begin{array}{l}\text { Perturba- } \\
\text { ción }+\end{array}$ & $\begin{array}{c}\text { Pendien- } \\
\text { te }\end{array}$ & Vegetación de cada "grupo" \\
\hline 5 & 1 & 19.05 & & $\mathrm{P}$ & $\begin{array}{l}\text { Selva baja caducifolia con Lysiloma microphylla y } \\
\text { Bursera spp. }\end{array}$ \\
\hline 1 & 2 & 22.22 & & $\mathrm{P}$ & \\
\hline 10 & 3 & 30.0 & & $\mathrm{~L}$ & \\
\hline 7 & 4 & 28.57 & & $\mathrm{~L}$ & Matorral nerturbado con Acacia cochliacantho \\
\hline & $\begin{array}{l}5 \\
6\end{array}$ & $\begin{array}{l}46.15 \\
29.41\end{array}$ & + & $\begin{array}{l}\mathrm{L} \\
\mathrm{L}\end{array}$ & $\begin{array}{l}\text { Matorral perturbado con Acacia cochliacantha } \\
\text { Selva baja caducifolia con Lysiloma microphylla y }\end{array}$ \\
\hline 10 & $\begin{array}{l}7 \\
8\end{array}$ & $\begin{array}{l}22.22 \\
31.58\end{array}$ & & $\mathrm{~L}$ & $\begin{array}{l}\text { Bursera spp. }\end{array}$ \\
\hline 3 & 9 & $\begin{array}{l}31.58 \\
26.67\end{array}$ & & $\mathrm{P}$ & \\
\hline 10 & 10 & 35.29 & & $\mathrm{P}$ & \\
\hline 7 & 11 & 42.86 & + & $\mathrm{P}$ & Cardonal y tetechera con Stenocereus weberi y Neo- \\
\hline & 12 & 63.64 & + & $\mathrm{P}$ & buxbaumia tetetzo \\
\hline 9 & 13 & 43.48 & + & $\mathrm{P}$ & Selva baja mixta con cardones y tetechos \\
\hline & 14 & 41.67 & + & $\mathrm{P}$ & Matorrales perturbados con Amphipterygium ads- \\
\hline & 15 & 75.0 & + & $\mathrm{L}$ & tringens (14) y Acacia cochliacantha (15-18) \\
\hline 6 & 16 & 71.43 & + & $\mathrm{L}$ & \\
\hline & 17 & 77.78 & + & $\mathrm{L}$ & \\
\hline & 18 & 61.54 & + & $\mathrm{L}$ & \\
\hline & 19 & 30.0 & & $\mathrm{P}$ & Selva baja caducifolia con Bursera spp., Cyrtocar- \\
\hline 4 & 20 & 42.86 & + & $\mathrm{P}$ & pa procera, Ceiba parvifolia \\
\hline & 21 & 25.0 & & $\mathrm{P}$ & \\
\hline & 22 & 18.18 & + & $\mathrm{P}$ & \\
\hline 8 & 23 & 35.71 & & $\mathrm{~L}$ & \\
\hline 3 & 24 & 35.29 & & $\mathrm{~L}$ & \\
\hline & 25 & 53.33 & + & $\mathrm{L}$ & Quiotillal con Escontria chiotilla \\
\hline & 26 & 62.50 & & $\mathrm{P}$ & Tetecheras con Neobuxbaumia tetetzo \\
\hline 2 & 27 & 60.0 & & $\mathrm{P}$ & \\
\hline & 28 & 50.0 & & $\mathrm{P}$ & \\
\hline & 29 & 57.10 & & $\mathrm{P}$ & \\
\hline 7 & 30 & 50.0 & & $\mathrm{~L}$ & \\
\hline 8 & 31 & 25.0 & + & $\mathrm{L}$ & Selva baja caducifolia con Bursera spp., Cyrtocar- \\
\hline 4 & 32 & 33.33 & & $\mathrm{P}$ & pa procera y Ceiba parvifolia \\
\hline & 33 & 33.33 & + & $\mathrm{L}$ & Matorral de Ipomoea wolcottiana \\
\hline 1 & 34 & 12.15 & + & $\mathrm{P}$ & \\
\hline & 35 & 42.86 & + & $\mathrm{P}$ & Matorral de Ipomoea wolcottiana con Acacia \\
\hline 5 & 36 & 42.86 & + & $\mathrm{P}$ & cochliacantha \\
\hline 5 & 37 & 71.43 & + & $\mathrm{P}$ & $\begin{array}{l}\text { Matorral de transición con Acacia subangulata y } \\
\text { Parthenium tomentosum }\end{array}$ \\
\hline
\end{tabular}

${ }^{*}$ Con respecto al total de especies presentes en cada censo.

${ }^{* *} \mathrm{P}=$ pronunciada.

$\mathrm{L}=$ ligera.

El censo 30 correspondería a una tetechera (grupo 2) pero por la presencia de un individuo de $S$. weberi es ubicado en otro grupo. Los censos 23 y 31 sufren del mismo fenómeno ya que en realidad presentan características fisonómicas y florísticas similares a los grupos 3 y 4 (selva baja caducifolia). Para el censo 25 la clasificación errónea se 


\section{ANÁLISIS DE LA VEGETAGIÓN DE TEHUACÁN-CUICATLÁN}

produce por la presencia de Neobuxbaumia tetetzo que lo ubica dentro de las tetecheras, siendo en realidad desde el punto de vista fisonómico y cuantitativo lo que Miranda (1948) denominó un quiotillal cuya especie característica es Escontria chiotilla.

Por otro lado, una serie de censos $(5,11,12,13)$ que se realizaron en sitios que tienen elementos combinados de diferentes tipos de vegetación fueron ubicados en sus propios grupos. Asimismo, en el grupo 5 quedaron ubicados 3 censos que demarcan los límites extremos del gradiente seguido y que aunque corresponden a selva baja caducifolia (censo 1) y matorrales (censos 36 y 37 ), comparten tres especies que los hacen únicos. Estas son: Acacia subangulata, Yucca periculosa y Beaucarnea gracilis. Las dos últimas especies sólo se encuentran en estos censos extremos que se localizan por arriba de los $1400 \mathrm{msnm}$.

El análisis de componentes principales (Fig. 4) produce un arreglo de los censos

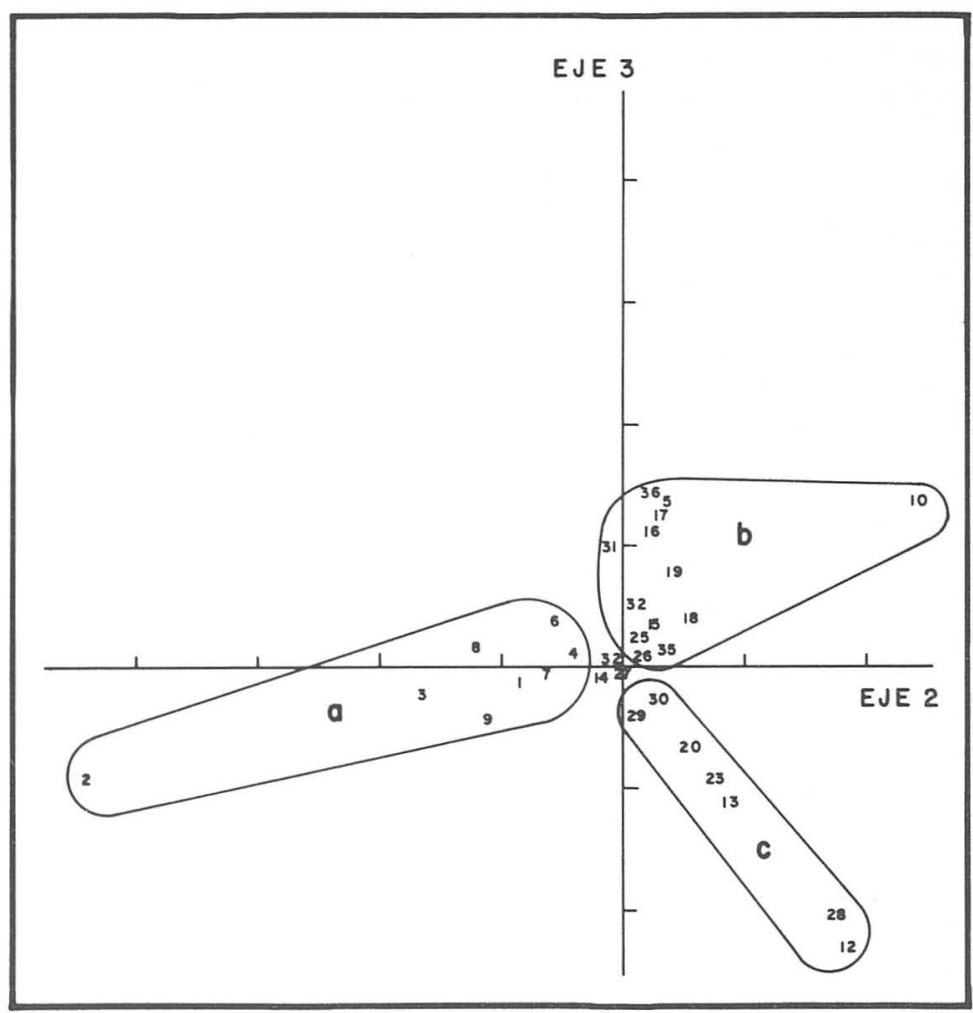

Fig. 4. Grupos de censos separados por el Análisis de Componentes Principales en función de los valores de dominancia de las especies; a) Lysiloma microphylla; b) Acacia cochliacantha; c) Cercidium plurifoliolatum. (Sólo se muestran los censos claramente separados por el análisis). 
que en algunos casos confirma lo establecido por el análisis de clasificación y en otros lo modifica. Los ejes que hacen la separación más clara de censos son el 2 y 3 y ésta es la combinación que se procedió a interpretar. Se puede observar que este análisis produce, fundamentalmente, la separación de los censos en 3 grupos en función de los valores de dominancia de las especies. Un grupo de censos (1-4, 6-9) cuya especie con altos valores de dominancia es Lysiloma microphylla; otro grupo (12, 13, 20, 23, 28, 30) donde la especie con más altos valores de dominancia es Cercidium plurifoliolatum; y por último el grupo de censos altamente perturbados (15-18) con otros que la clasificación no había ubicado con ellos (5, 10, 19, 25, 31, 32, 35, 36) donde Acacia cochliacantha tiene valores altos de dominancia. Sin embargo, puede decirse que este análisis no resultó lo satisfactorio que se esperaba, probablemente porque la heterogeneidad de los datos fue muy grande (ver Lambert y Dale, 1964).

La ordenación por promediación recíproca, tanto cualitativa como cuantitativa, pareció sufrir aún más los efectos de la heterogeneidad ya que varios de los ejes permitieron únicamente separar a unos cuantos censos del resto (Fig. 5). El primer eje de este análisis permitió confirmar que tanto el censo 1 como el 36 y 37 marcan extremos no solamente físicos sino también flurísticos, que los hacen marcadamente diferentes de los demás; hecho que había sido detectado también por el análisis de clasificación.

\section{DISCUSIÓN}

Los resultados aquí presentados muestran que de los 10 grupos producidos por la clasificación podemos definir 6 " grupos" que corresponderían a tipos de vegetación descritos con un punto de vista fisonómico-descriptivo. Ellos son:

a) Selva baja caducifolia o bosque tropical caducifolio

b) Tetecheras de Neobuxbaumia tetetzo

c) Cardonal de Stenocereus weberi

d) Quiotillal de Escontria chiotilla

e) Matorral con Acacia cochliacantha

f) Matorral con Ipomoea wolcottiana y A. cochliacantha

Estos tipos de vegetación habían sido mencionados para la zona por Miranda (1948). Sin embargo, la aplicación de métodos de muestreo objetivos y cuantitativos, así como la utilización de métodos de análisis multivariado, permitió detectar la variación de la vegetación con mucho más detalle. Por ejemplo: la selva baja caducifolia (bosque de hojas medianas caedizas, sensu Miranda, 1948) se presenta, no como una comunidad uniforme y homogénea sino conformada por dos variantes que se encuentran una en cada valle. La dominada por Lysiloma microphylla se localiza en el extremo sureste del área muestreada, en las inmediaciones de Cuicatlán y Dominguillo y fue detectada muy claramente tanto por el análisis de clasificación como por el de componentes principales. La otra variante de esta selva se localiza desde los alrededores de Teotitlán, Oax., hasta Zinacatepec, Pue., dominada por Bursera spp. y Cyrtocarpa procera y fue detectada por el análisis de clasificación. De acuerdo con Rzedowski (1978), el bosque tropical caducifolio (selva baja caducifolia, sensu Miranda y Hernández X., 1963) se distribuye en áreas donde la temperatura mínima es superior a los $0{ }^{\circ} \mathrm{C}$. Si ubicamos los censos que corresponden a la selva baja con Bursera spp. y C. procera de la zona de estudio en un mapa de temperaturas mínimas (Fig. 6), se observa que el extremo norte de su distribución se encuentra entre las isolíneas de 3 y $4{ }^{\circ} \mathrm{C}$, lo cual corrobora dicha aseveración. 


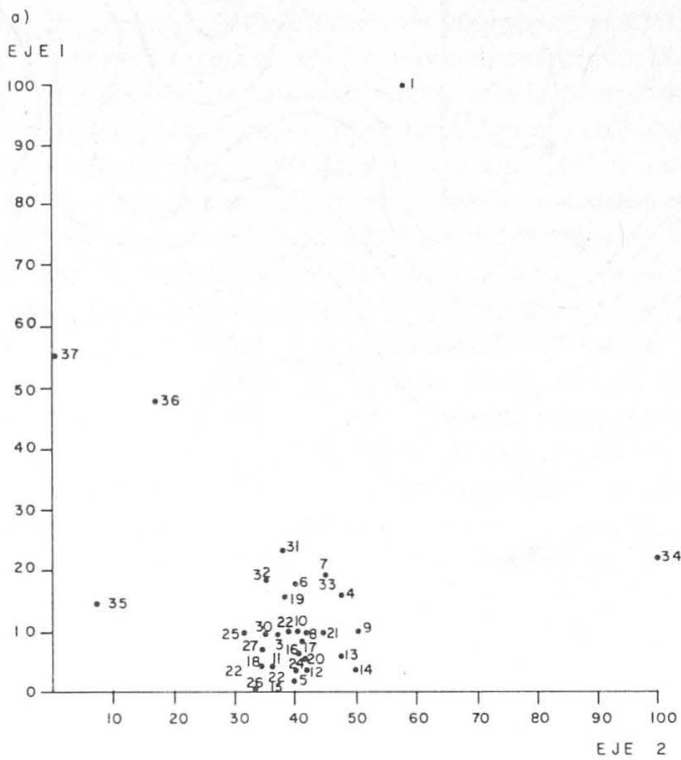

b)

EJE I

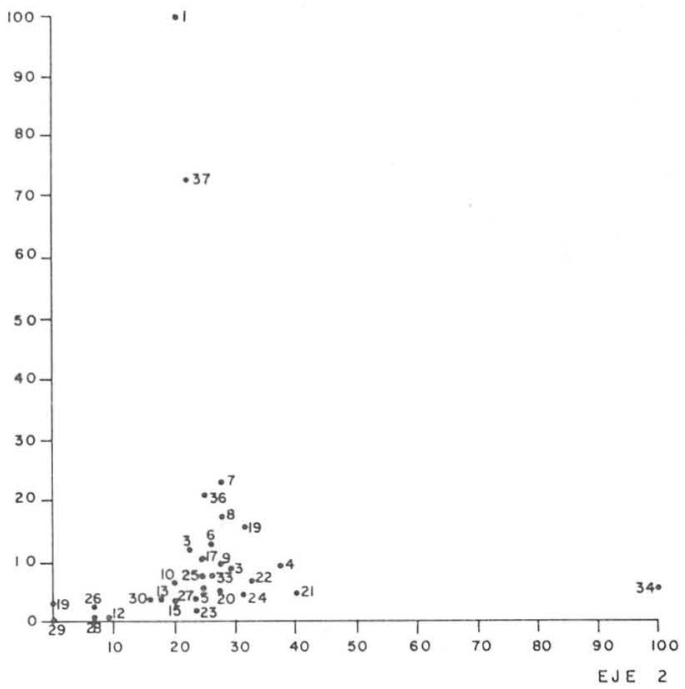

Fig. 5. Promediación recíproca por censos de la vegetáción arbórea mostrando la escasa dispersión de los censos a lo largo de los ejes. a) Cualitativa; b) Cuantitativa. 


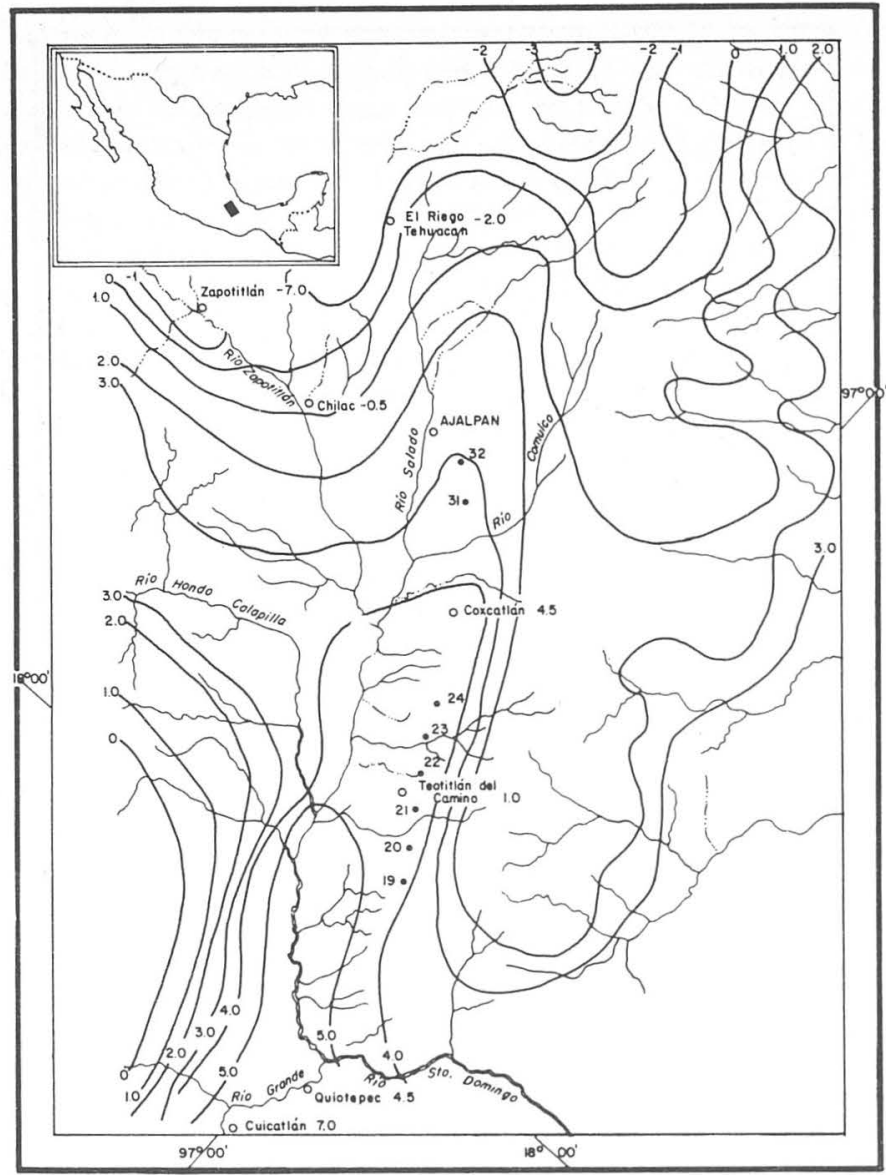

Fig. 6. Mapa de isolíneas de temperaturas mínimas. Los censos marcados presentan vegetación de selva baja caducifolia con Bursera spp. y Cyrtocarpa procera (ver texto). (Tomado de Byers, 1967).

En su tratamiento del bosque tropical caducifolio, Rzedowski (1978) citando a Miranda, asevera que la perturbación de esta comunidad produce una sucesión que pasa por un período dominado por Acacia cochliacantha, para después pasar a una comunidad de Ipomoea wolcottiana y con el tiempo restablecer la comunidad clímax (Rzedowski 1978, p. 202). En el presente trabajo se tienen censos dominados por cada una de las especies y algunos casos en que ambas se presentan en la misma muestra (ver tabla 1). Sería muy interesante seguir el patrón de variación de estas comunidades a través del tiempo para implementar una teoría más objetiva y fundada sobre este fenómeno sucesional del bosque tropical caducifolio. Rzedowski (1978) menciona que en condiciones no perturbadas, el elemento espinoso no tiene nucha importancia en dicha comunidad. Pen- 


\section{ANÁLISIS DE LA VEGETACIÓN DE TEHUAGÁN-GUICATLÁN}

sando en términos sucesionales, esperaríamos encontrar que cuando este bosque es perturbado drásticamente el porcentaje de elementos espinosos aumente notablemente y que al pasar por sus diferentes estados serales vaya disminuyendo tal porcentaje. En un contexto ecológico, el aumento de este porcentaje con la perturbación podría deberse a una respuesta al ambiente más seco que se produce al recibirse mayor insolación por la ausencia de árboles con copas que la intercepten. En un contexto de manejo, la perturbación producida por un sobrepastoreo de las comunidades en cuestión, traería como consecuencia el aumento del porcentaje de especies espinosas. Los resultados que se resumen en la tabla 1 muestran que las comunidades pertubadas con $A$. cochliacantha presentan un porcentaje no menor al $45 \%$ y hasta de casi $78 \%$ de especies espinosas. Los matorrales de $I$. wolcottiana y los de $I$. wolcottiana con $A$. cochliacantha tienen valores por debajo del $45 \%$ y hasta de $33 \%$ (con excepción de un caso extremo de $12.5 \%$ ). La selva baja caducifolia con sus dos variantes presenta en la gran mayoría de los casos, porcentajes inferiores al 33\%. Estos resultados sugieren que en efecto, el patrón sucesional del bosque tropical caducifolio presenta variaciones florísticas y ecológicas que merecen estudios más detallados.

Las tetecheras, agrupaciones de cactáceas columnares que en ocasiones son tan densas que impiden la coexistencia de árboles (Miranda, 1948, Rzedowski, 1978), fueron claramente detectadas por la clasificación en función de la ausencia de una especie: Bursera submoniliformis. Esta especie es un árbol que, en general, tiene una copa muy abierta y por lo tanto una cobertura muy amplia. Tomando en cuenta que las tetecheras ocupan un área en cuyos límites encontramos la selva baja caducifolia con diversas especies de Bursera y donde $B$. submoniliformis es muy característica, su ausencia en las tetecheras puede tener un significado biológico interesante, sobre todo si consideramos que otras especies de Bursera más pequeñas (i.e. B. aptera) sí se encuentran coexistiendo con Neobuxbaumia tetetzo. Sería interesante explorar con detalle las relaciones de competencia que ocurren en las tetecheras y definir si este tipo de interacciones bióticas desempeñan un papel importante en el establecimiento de ciertas especies como $B$. submoniliformis.

Desde un punto de vista estricto sólo un censo (12) puede considerarse como un cardonal y otro (25) como un quiotillal. El cardonal presenta un alto número de elementos de la selva baja pero con una marcada dominancia del cardón (Stenocereus weberi). El quiotillal, caracterizado por Escontria chiotilla se encuentra generalmente en terrenos profundos y cercanos a los poblados (Miranda, 1948). Para el caso particular de este censo, la dificultad de acceso a las laderas propició que el muestreo se realizara en terrenos planos dando como consecuencia la aparición de esta comunidad rompiendo aparentemente la continuidad de las tetecheras que se establecen sobre las laderas.

Miranda (1948) menciona que el izotal, comunidad caracterizada por Yucca periculosa y que se presenta en las cercanías de Tehuacán, desaparece bruscamente por abajo de los $1500 \mathrm{msnm}$. El presente trabajo muestra que, en efecto, solo dos censos cercanos a Tehuacán (36 y 37) y que se encuentran por arriba de los 1400 msnm presentan .a esta especie en la comunidad aunque sin formar un izotal típico. Sin embargo, justo en el otro extremo de la región a $160 \mathrm{~km}$ de distancia (censo 1) y también por arriba de los $1400 \mathrm{msnm}$, pero en una comunidad totalmente diferente, encontramos nuevamente a $Y$. periculosa. Este hecho sugiere una respuesta de esta especie a condiciones del ambiente físico que merece investigación más detallada.

Los resultados del presente trabajo ponen en duda la ocurrencia de un tipo de vegetación que Miranda (op. cit.) describió para la zona y que denominó "'bosque bajo, 
espinoso, de hojas pequeñas y caedizas" dominado por Cercidium plurifoliolatum. Aunque el análisis de componentes principales separa un grupo de censos donde C. plurifoliolatum es una especie con altos valores de dominancia (Fig. 4), tales censos, por sus características florísticas, fisonómicas y cuantitativas han sido definidos en el presente trabajo, como selva baja caducifolia (20,23), cardonal (12), cardonal con selva baja (13) y tetecheras $(28,29,30)$. Este hecho nos muestra que, más que poder definir un tipo de vegetación dominado por C. pluriofoliolatum como lo había hecho Miranda (1948) desde un punto de vista fisonómico-descriptivo, podemos decir que esta especie presenta una gran plasticidad ya que puede coexistir con un alto número de especies diferentes y presumiblemente, en condiciones ambientales diversas.

Si consideramos, a manera de comparación, los alcances de cada uno de los métodos de análisis utilizados, el que proporcionó más herramientas útiles para el análisis fue el de clasificación (CENOSIS 2), seguido por el de ordenación por componentes principales y, por último, el de promediación recíproca. Los resultados sugieren que la heterogeneidad del total de censos fue muy grande, ya que los ejes de ordenación se centraron sobre la detección de la individualidad de un número reducido de ellos, sobre todo en el caso de la promediación recíproca en el cual los primeros cuatro ejes sólo separaron unos cuantos censos del resto. Estos resultados concuerdan con lo señalado por diversos autores (Lambert y Dale, 1964; Greig-Smith et al., 1967; Beals, 1973; Goodall, 1978) en cuanto a que en un nivel alto de variación vegetacional el método de clasificación proporciona un arreglo más satisfactorio que la ordenación.

La aplicación de estas técnicas de análisis al estudio de la vegetación puso de manifiesto que la clasificaєión y la ordenación no son métodos opuestos sino que son complementarios tal y como había sido señalado con anterioridad. De hecho, se hizo evidente que la ordenación puede indicar la necesidad de reubicar censos mal agrupados en la clasificación y que la ordenación se puede utilizar como una revisión y extensión de ella (ver Greig-Smith et al., 1967).

Con base en este trabajo podemos decir que la variación de la vegetación en la zona de estudio responde no sólo a una interrelación de factores ambientales (clima, suelo, sustrato), sino que también, y de manera muy importante, a la perturbación humana que afecta fuertemente la estructura y composición de las comunidades. Aparentemente, también, interacciones bióticas (i.e. competencia), pueden desempeñar un papel relevante. Asimismo, el presente trabajo muestra que la vegetación arbórea de la porción estudiada del Valle de Tehuacán-Cuicatlán es más heterogénea y variable que lo que estudios fisonómico-descriptivos anteriores habían demostrado (Shreve, 1942; Miranda, 1948; Smith, 1965). Se sugiere por lo tanto, la realización de este tipo de estudios, ya que permiten determinar de una manera objetiva grupos de especies con semejantes requerimientos ecológicos, patrones de variación (i.e. sucesionales), así como el comportamiento de especies individuales. De esta forma se facilita la elaboración de hipótesis susceptibles de ser investigadas con estudios más detallados o con estudios experimentales para conocer los factores que determinan la distribución y abundancia de las especies.

Algunas cuestiones a considerar para futuras investigaciones serían:

a) Intensificar los muestreos en las áreas ocupadas por "grupos" producidos por la clasificación y que presentan cierta homogeneidad (p. ej. el "grupo" de Lysiloma, el de tetecheras, etc.) para realizar ordenaciones parciales que nos permitan explorar con más detalle la variación de la vegetación y explicarla ecológicamente, ya que la información producida por la ordenación dentro de los "gru- 


\section{ANÁLISIS DE LA VEGETACIÓN DE TEHUACÁN-CUICATLÁN}

pos" no se puede obtener considerando subjetivamente los datos (ver GreigSmith et al., 1967).

b) Investigar con detalle la biología de ciertas especies que por sus respuestas, tanto a factores ecológicos como de perturbación humana las hacen de singular importancia en las comunidades de la zona (p. ej. Stenocereus weberi, Lysiloma microphylla, Bursera submoniliformis, Yucca periculosa, Cercidium plurifoliolatum).

c) Explorar sistemáticamente el patrón sucesional de la selva baja caducifolia empezando con la cuantificación, en sitios predeterminados, de aspectos sencillos como los aquí referidos (i.e. espinosidad, especies secundarias y manejo).

\section{AGRADECIMIENTOS}

Agradecemos al doctor Rodolfo Dirzo (Instituto de Biología, UNAM) por la revisión crítica del manuscrito y al ingeniero Víctor Jaramillo Villalobos, Director General de COTECOCA, SARH, por haber brindado las facilidades para la elaboración material (mecanografía y figuras) del trabajo.

\section{BIBLIOGRAFÍA}

ANDERSON, D.J. 1965. Classification and ordination in vegetation science: controversy over a non-existent problem? J. Ecol.:521-526.

ANÓNIMO, 1981. Atlas nacional del medio físico. Secretaría de Programación y Presupuesto.

AUSTIN, M.P. 1972. Models and analysis of descriptive vegetation data. pp. 61-86. En: J.N. Jeffers (Ed.) Mathematical models in ecology. Blackwell Scientific Publications, Oxford.

AUSTIN, M.P. y L. ORLOCI. 1966. Geometric models in ecology. II. An evaluation of some ordination techniques. J. Ecol. 54:217-227.

BEALS, E.W. 1973. Ordination: mathematical elegance and ecological naiveté. J. Ecol. 61:23-35.

BRAY, J.R. y J.T. CURTIS. 1957. An ordination of the upland forest communities of southern Wisconsin. Ecol. Monogr. 27:325-349.

BROWN, R.T. y J.T. CURTIS. 1952. The upland conifer-hardwood forests of northern Wisconsin. Ecol. Monogr. 22:217-234.

BYERS, D.S. 1967. Climate and hydrology. pp. 48-65. En: D.S. Byers (Ed.) The Prehistory of the Tehuacan Valley. Vol. 1. Enviroment and Subsistence. University of Texas Press, Austin.

COTTAM, G. y J.T. CURTIS. 1956. The use of distance measures in phytosociological sampling. Ecology 37:451-460.

CURTIS, J.T. 1955. A prairie continuum in Wisconsin. Ecology 36:558-566.

CURTIS, J.T. y R.P. McINTOSH. 1951. An upland forest continuum in the prairieforest region of Wisconsin. Ecology 32:476-496.

EZCURRA, E. 1978. Principal components analysis of the understory vegetation of Treborth Woods: the effect of data transformations. M. Sc. Thesis, University College of North Wales, Bangor.

GARCIA, E. 1973. Modificaciones al sistema de clasificación climática de Köeppen (para adaptarlo a las condiciones de la República Mexicana). Universidad Nacional Autónoma de México, México.

GOODALL, D.W. 1953. Objective methods for the classification of vegetation. I. The use of positive interspecific correlation. Austral. J. Bot. 1:39-63. 
GOODALL, D.W. 1954. Objective methods for the classification of vegetation. III. An essay in the use of factor analysis. Austral. J. Bot. 2:304-324.

GOODALL, D.W. 1970. Statistical plant ecology. Ann. Rev. Ecol. Syst. 1:99-124.

GOODALL, D.W. 1978. Numerical classification. pp. 247-286. En: R.H. Whittaker (Ed.) Classification of plant communities. Dr. W. Junk, The Hague.

GREIG-SMITH, P., M.P. AUSTIN y T.C. WHITMORE. 1967. The application of quantitative methods to vegetation survey. I. Association analysis and principal component ordination of rain forest. J. Ecol. 55:483-503.

HILL, M.O. 1973. Reciprocal averaging: an eigenvector method of ordination. J. Ecol. 61:237-244.

KERSHAW, K.A. 1973. Quantitative and dynamic plant ecology. Edward Arnold, London.

LAMBERT, J.M. y M.B. DALE. 1964. The use of statistics in phytosociology. Advances. Ecol. Res. 2:59-99.

LAMBERT, J.M. y W.T. WILLIAMS. 1966. Multivariate methods in plant ecology. VI. Comparison of information-analysis and association-analysis. J. Ecol. 54:634-664.

LÓPEZ RAMOS, E. 1974. Carta geológica del estado de Oaxaca. Instituto de Geología, UNAM, México.

LÓPEZ RAMOS, E. 1979. Carta geológica de los estados de Tlaxcala y Puebla. Instituto de Geología, UNAM, México.

MAAREL, E. VAN DER. 1969. On the use of ordination models in phytosociology. Vegetatio 19:21-46.

McINTOSH, R.P. 1967. The continuum concept of vegetation. Bot. Rev. 33:130-187. MIRANDA, F. 1948. Datos sobre la vegetación de la cuenca alta del Papaloapan. Anales Inst. Biol. Univ. Nac. México 19 Ser. Botánica: 333-364.

MIRANDA, F. y E. HERÁNDEZ X. 1963. Los tipos de vegetación de México y su clasificación. Bol. Soc. Bot. México 28:29-178.

MUELLER-DOMBOIS, D. y H. ELLENBERG. 1974. Aims and methods of vegetation ecology. John Wiley, New York.

ORLOCI, L. 1966. Geometric models in ecology. I. The theory and application of some ordination methods. J. Ecol. 54:193-215.

RZEDOWSKI, J. 1978. Vegetación de México. Editorial Limusa, México.

SHREVE, F. 1942. The desert vegetation of North America. Bot. Rev. 8:195-245.

SMITH, E.C. 1965. Flora, Tehuacan Valley. Fieldiana Bot. 31:101-143.

WEBB, L.J., J.G. TRACEY, W.T. WILLIAMS y G.N. LANCE. 1967. Studies in the numerical analysis of complex rain-forest communities. I. A comparison of methods applicable to site/species data. J. Ecol. 55:171-191.

WHITTAKER, R.H. 1970. The population structure of vegetation. pp. 360-380. En: R.P. McIntosh (Ed.) Phytosociology. Dowden, Hutchinson \& Ross, Stroudsburg. WHITTAKER, R.H. 1975. Communities and Ecosystems. McMillan, New York. WILLIAMS, W.T. y J.M. LAMBERT. 1959. Multivariate methods in plant ecology. I. Association-analysis in plant communities. J. Ecol. 47:83-101.

WILLIAMS, W.T. y J.M. LAMBERT. 1961. Multivariate methods in plant ecology. III. Inverse association-analysis. J. Ecol. 49:717-729.

WILLIAMS, W.T., J.M. LAMBERT y G.N. LANCE. 1966. Multivariate methods in plant ecology. V. Similarity-analysis and information-analysis. J. Ecol. 54:427-445. 